\title{
Aportes al estudio de las relaciones entre memoria episódica y Teoría de la Mente
}

\section{Contributions to the study of the relations between episodic memory}

\author{
and Theory of Mind
}

\author{
María José Aguilar ${ }^{1}$, Lucía Agulla $^{2}$, Andrea Said $^{3}$ y Marcela López ${ }^{4}$ \\ ${ }^{1}$ Doctora en Psicología. Becaria postdoctoral del Consejo Nacional de Investigaciones Científicas \\ y Técnicas (CONICET). E-mail: majoaguilar1@gmail.com \\ ${ }^{2}$ Licenciada en Psicología. Becaria de la Universidad Nacional de Mar del Plata. \\ E-mail: luciaagulla@gmail.com \\ ${ }^{3}$ Licenciada en Psicología. Becaria doctoral del Consejo Nacional de Investigaciones Científicas \\ y Técnicas (CONICET). E-mail: andrea_said@hotmail.com \\ ${ }^{4}$ Doctora en Psicología. Licenciada en Ciencias Biológicas. Docente exclusiva regular de la Fa- \\ cultad de Psicología, Universidad Nacional de Mar del Plata. E-mail: mclopez@mdp.edu.ar \\ Instituto de Psicología Básica, Aplicada y Tecnología (IPSIBAT). \\ Consejo Nacional de Investigaciones Científicas y Técnicas (CONICET) \\ Facultad de Psicología, Universidad Nacional de Mar del Plata. \\ Mar del Plata, Prov. de Buenos Aires, Argentina.
}

\section{Resumen}

La teoría de la mente emerge en el desarrollo con anterioridad a la memoria episódica, posibilitando a través de la capacidad de metarrepresentación el desdoblamiento y viaje mental que ella misma implica. Para la evaluación de los procesos se administraron las Historias Extrañas de Happé, el Test de las miradas, el Test de Aprendizaje Verbal España-Complutense y una tarea experimental a 20 mujeres con diagnóstico de Síndrome de Turner y sus respectivos controles. Los resultados indican dificultades en ambos procesos cognitivos y correlaciones entre la teoría de la mente e indicadores de memoria episódica de contenido y de la fuente. Se hipotetiza que la anatomía cerebral atípica, propia de esta población, traería problemas en el desarrollo de la teoría de la mente y esto, a su vez, podría asociarse con dificultades en los mecanismos de metarrepresentación y reexperimenta- ción subjetiva de vivencias que la memoria episódica implica. Los resultados obtenidos permiten una mayor comprensión del perfil neuropsicológico de las mujeres con diagnóstico de Síndrome de Turner y podrían servir de insumo teórico para el diseño de estrategias clínicas y psicoeducativas que tiendan a fomentar, en esta población, las habilidades de teoría de la mente y memoria episódica.

Palabras clave: Memoria episódica; Teoría de la mente; Metarrepresentación; Perfil neuropsicológico; Síndrome de Turner.

\section{Abstract}

The present study explores the relations between the cognitive and affective processes of theory of mind and verbal episodic memory in women diagnosed with Turner Syndrome, a chromosomal disorder caused by a missing or incomplete $\mathrm{X}$ chromosome in females. Women with this diagnostic present an 
atypical cerebral morphology affecting frontal and temporoparietal zones. These areas match the neuroanatomic substratum shared by both theory of mind and episodic memory. The concept of theory of mind refers to the ability to anticipate others' social behavior through the attribution and understanding of mental entities such as desires, beliefs, emotions and intentions. It is a complex skill that involves not only the mental representation of something that cannot be observed directly, but also the decentration of one's own perspective and the use of these skills to predict behaviors. This ability is composed by two different processes: a cognitive theory of mind that refers to the ability to make inferences about desires, beliefs and intentions of other people; and an affective theory of mind, related to the ability to infer others' emotions, understanding the affective mental states and adopting the point of view of the other person, without experiencing these emotions. In the particular case of women diagnosed with Turner Syndrome, there is evidence supporting the hypothesis that this population presents general difficulties in this ability, showing a greater deficit in cognitive theory of mind. On the other hand, episodic memory consists of the memory that one has of past experiences. This memory system is a psychological process of paramount importance for human beings, since it enables the remembrance of something that has happened a long time ago. It allows the person to re-experience events that occurred earlier in his or her life, involving the ability to generate meta-representational comments on how knowledge was obtained. This way, people revive, through self-awareness, previous experiences and also project similar experiences to the future. Episodic memory can be divided into the memory about the occurrence of an event (item memory) and the memory of the phenomenological context of the event, which involves the handling of spatial, temporal, emotional and perceptual information (source memory). It is considered that theory of mind has an earlier development than episodic memory, enabling through the capacity of meta-representation the unfolding and mental journey that episodic memory implies. In order to evaluate these processes, a battery of four tests was administered to 20 women diagnosed with Turner Syndrome and their respective controls: Happé Strange Stories, the Reading the Mind in the Eyes Test, the Spain-Complutense Verbal Learning Test and an experimental task. Results indicate difficulties in both cognitive processes, as well as correlations between theory of mind and indicators of item and source memory. A possible hypothesis could argue that the atypical cerebral anatomy of women diagnosed with Turner Syndrome would result in theory of mind deficits, and these in turn could be associated with difficulties in the capacities of meta-representation and subjective re-experimentation of past events needed by episodic memory. These results enable a better comprehension of the neuropsychological profile of women diagnosed with Turner Syndrome and could serve as a theoretical input for the design of clinical and psychoeducational strategies that tend to promote theory of mind and episodic memory in this population.

Keywords: Episodic Memory; Theory of Mind; Meta-representation; Neuropsychological profile; Turner Syndrome.

\section{Introducción}

El Síndrome de Turner (ST) es uno de los trastornos cromosómicos no heredables con mayor incidencia (1:2500 niñas nacidas vivas), y está determinado por la deleción total o parcial del cromosoma $\mathrm{X}$ en el sexo femenino (Van den Hoven, Roos-Hesselink y Timmermans, 2017). Dentro de las características físicas más frecuentes se encuentran estatura baja, hipogonadismo debido a disgenesia gonadal, cuello alado, enfermedad cardiovascular congénita, anomalías renales y desórdenes en el desarrollo neurológico (Klein et al., 2018). 
Las mujeres con diagnóstico de ST presentan perfiles cognitivos y sociales distintivos, determinados no solo por la falta de expresión génica del cromosoma $X$, sino también por las interacciones entre este déficit y otros factores genéticos, biológicos y ambientales, existiendo variaciones tanto a nivel poblacional, como a nivel individual (Anaki, Zadikov-Mor, Gepstein y Hochberg, 2016). En cuanto a las características neuroanatómicas del cerebro, se ha reportado un mayor volumen general intracraneal expresado en una mayor cantidad de sustancia blanca y gris en los hemisferios cerebrales (lóbulos frontal, temporales, parietales y occipital) y el sistema límbico (amígdala e hipocampo), así como déficit en el funcionamiento de las vías neuronales entre las regiones frontales y temporoparietales (Granger, Zurada, Zurada-Zielińska, Gielecki y Loukas, 2016; Marzelli, Hoeft, Hong y Reiss, 2011).

Estas características traen aparejado un perfil cognitivo particular marcado por una disfunción en las funciones ejecutivas; se ven afectados el control inhibitorio, la flexibilidad cognitiva, la memoria de trabajo y la toma de decisiones (Mauger et al., 2018). Asimismo, se han observado problemas en habilidades visuoespaciales y dificultades para generar y/o comprender conceptos abstractos, mantener la atención activa, y para planificar y llevar a cabo tareas complejas (Lepage, Dunkin, Hong y Reiss, 2011; Vargo, Klabunde, Hong y Reiss, 2017). Las funciones cognitivas afectadas son esenciales para responder adaptativamente a las demandas cambiantes del medio ambiente, lo cual les genera dificultades en su funcionamiento diario.

Por otro lado, las problemáticas que las mujeres con diagnóstico de ST presentan para interactuar con sus pares son una constante durante su desarrollo y son consecuencia de dificultades en el funcionamiento global psicosocial. La literatura describe problemas para conformar y mantener relaciones interpersonales, sociales e íntimas (Hong, Dunkin y Reiss, 2011; Lesniak-Karpiak, Mazzocco y
Ross, 2003; Sartori y López, 2016; Sartori, Urquijo, López, Said y Alchieri, 2015), y menores posibilidades de alcanzar una forma de vida autónoma y logros profesionales compatibles con su nivel educativo (Anaki et al., 2016). Mazzocco (2006) considera que los problemas en el funcionamiento social podrían ser secundarios a disfunciones en otras áreas como las encargadas del procesamiento afectivo/facial o del funcionamiento ejecutivo, como así también a un repertorio limitado de comportamientos sociales. Hong et al. (2011) indican que si bien la disfuncionalidad social suele estar asociada a una multiplicidad de factores físicos y psicológicos (cariotipo, baja talla, desajustes hormonales, ansiedad social, baja autoestima, entre otros), ninguno de ellos explica completamente las dificultades sociales observadas, constituyéndose en una de las áreas de mayor vulnerabilidad.

Una de las líneas de investigación que buscan explicar las alteraciones en el funcionamiento social de esta población lo constituye el estudio de la teoría de la mente (TdM). El concepto de TdM, según Rivière (1996), se refiere a la capacidad de anticiparse a la conducta social de los demás a través de la adscripción y comprensión de entidades mentales como los deseos, creencias, emociones e intenciones. Se trata de una habilidad compleja que implica no solo la representación mental de algo que no es posible de ser observado directamente, sino también la descentración de la perspectiva propia y el empleo de estas habilidades para predecir comportamientos (Tirapu-Ustárroz, Pérez-Sayesa, Erekatxo-Bilbaoa y Pelegrín-Valerob, 2007). Según Sodian (2005), la TdM es una etiqueta para los conceptos psicológicos de sentido común que se usan para atribuir estados mentales a uno mismo y a los demás (saber, pensar, sentir, querer).

Dichas características hacen de la TdM una habilidad imprescindible para un adecuado desenvolvimiento social ya que, sin la atribución de intenciones y deseos a los otros, resulta difícil establecer y sostener relaciones como 
la amistad, el compañerismo, la competitividad, resolver conflictos sociales y mantener intercambios comunicativos que requieren la interpretación de un sentido no literal (González-Cuenca, Barajas-Esteban, Linero-Zamorano y Quintana-García, 2008). La disfunción en cualquiera de estos procesos trae aparejadas consecuencias que dificultan la capacidad para comprender el entorno inmediato $\mathrm{y}$, por lo tanto, limitan un buen desarrollo social.

En relación con la TdM se distinguen dos procesos: una TdM cognitiva o fría, que refiere a la capacidad para realizar inferencias sobre los deseos, creencias e intenciones de otras personas, y una TdM afectiva o cálida, relacionada con la capacidad de inferir las emociones de los demás, comprendiendo los estados mentales afectivos y adoptando el punto de vista de la otra persona, sin necesidad de experimentar dicha emoción (Duval, Piolino, Bejanin, Eustache y Desgranges, 2011; Kalbe et al., 2010). Ambos procesos comparten un sustrato neuroanatómico, compuesto por el surco temporal superior, la amígdala y el cíngulo (Shamay-Tsoory, Harari, Aharon-Peretz y Levkovitz, 2010). Wang y Su (2013) indican que la TdM cognitiva se relaciona con la corteza prefrontal dorsomedial, mientras que la TdM afectiva, con la corteza prefrontal ventromedial.

En el caso particular de las mujeres con diagnóstico de ST, Aguilar, Zabala, López-Morales, Urquijo y López (2016) aportaron evidencias que avalan la hipótesis que señala que esta población presenta dificultades de carácter general en la TdM y un rendimiento diferencial en los aspectos cognitivos y afectivos, con mayores dificultades en los aspectos cognitivos.

Por otro lado, la literatura presenta un debate con respecto a la relación entre la TdM y otras funciones cognitivas, en el que se plantean dos perspectivas posibles. La primera considera a la TdM como un constructo independiente, dada la existencia de dobles disociaciones entre ella y otras funciones cognitivas (Hughes y Devine, 2015; Schneider,
Schumann-Hengsteler y Sodian, 2005). La segunda perspectiva propone que la TdM no consistiría en un módulo de carácter específico, sino general (Hughes y Devine, 2015; Moses, Carlson y Sabbagh, 2005), que involucra diferentes procesos bottom-up, como el procesamiento de emociones faciales y la detección del movimiento de ojos y cuerpo, y top-down, como la memoria episódica (ME) (Moreau, Viallet y Champagne-Lavau, 2013).

La ME refiere a un tipo de recuerdo que se tiene de experiencias pasadas. Este sistema de memoria constituye un proceso psicológico de primordial importancia para el ser humano, ya que permite que se recuerde algo que ha pasado tiempo atrás. Tulving (2002) considera la ME como la más elevada de las capacidades mentales, con un desarrollo ontogénico y filogenético tardío y probablemente exclusivo de la especie humana. Esta capacidad supone el funcionamiento conjunto de la corteza prefrontal y estructuras del lóbulo medial temporal, hipocampo y amígdala (Eichenbaum, 2017).

Una de las funciones principales de la ME consiste en facilitar predicciones para apoyar la capacidad de hacer nuevas inferencias y guiar la conducta adaptativa en el presente o en el futuro inmediato, en tanto es el único sistema de memoria que ofrece información espacial y temporalmente específica sobre experiencias únicas (Allen y Fortin, 2013; Schiller et al., 2015). Este sistema permite a la persona reexperimentar sucesos que ocurrieron anteriormente en su vida, involucrando la capacidad de generar comentarios metarrepresentacionales de cómo el conocimiento ha llegado a la mente (Solcoff, 2011). De esta manera, las personas reviven, a través de la conciencia autonoética, experiencias previas y también proyectan experiencias similares al futuro. Esta capacidad de recuperación posee características distintivas, por lo que es considerada exclusiva de la especie humana (Tulving, 2005).

Dentro del dominio de ME pueden distinguirse la memoria acerca de la ocurrencia 
de un evento (memoria de contenido o item memory) y la memoria del contexto fenomenológico del evento, que implica el manejo de información espacial, temporal, emocional y perceptual (memoria de la fuente o source memory) (Cooper, Greve y Henson, 2017).

En relación con los vínculos entre TdM y ME, Perner (2000) plantea la necesidad del desarrollo de la TdM para la formación de huellas episódicas, ya que la TdM posibilita la atribución del origen del conocimiento actual a la correspondiente experiencia pasada. A su vez, numerosos trabajos han propuesto que la TdM y la ME poseen mecanismos cognitivos comunes, como la conciencia autonoética, la autoproyección y el control de fuente (Buckner y Carroll, 2007; Lind, Williams, Raber, Peel y Bowler, 2013; Naito, 2003; Vázquez-Echeverría, 2015). Asimismo, estudios de neuroimagen han mostrado que estos dos procesos poseen áreas de activación cerebral compartidas. Algunas de las estructuras que comparten son la corteza prefrontal medial y posterior cingulada, el polo frontal, el giro frontal inferior, el lóbulo temporal medial (giro parahipocampal, amígdala), surco temporal superior, giro temporal medio y el giro angular (Moreau et al., 2013; Spreng y Mar, 2012). A su vez, numerosas investigaciones indican que dificultades en la capacidad de TdM traen aparejados problemas en la capacidad de comprender el entorno inmediato $\mathrm{y}$, en consecuencia, en el desarrollo de la ME, comprometiendo la capacidad de experimentar conscientemente acontecimientos personales que ocurrieron en lugares y momentos específicos, asociados con sentimientos particulares (Nandrino et al., 2014; Solcoff, 2011).

De acuerdo con lo anteriormente expuesto, el ST se presenta como un modelo pertinente para la exploración de las relaciones entre los procesos cognitivos y afectivos de TdM y la ME. Cabe destacar que los estudios reportan que esta población presenta dificultades de carácter general en la TdM, por lo que indagar las relaciones que establece con la $\mathrm{ME}$ verbal permitirá una mayor comprensión del perfil neuropsicológico de las mujeres con diagnóstico de ST. De esta manera, se intenta aportar un sustento teórico para el diseño futuro de intervenciones terapéuticas que mejoren la calidad de vida de las mujeres con este diagnóstico, basado en la relación entre ambos procesos.

\section{Método}

\section{Tipo de estudio}

El estudio que se presenta es de tipo no experimental, con diseño transversal, descriptivo correlacional, según la clasificación de Hernández-Sampieri, Fernández-Collado $\mathrm{y}$ Baptista-Lucio (2014).

\section{Participantes}

El universo de estudio está conformado por niñas, adolescentes y adultas con diagnóstico de ST de la ciudad de Mar del Plata y de la Ciudad Autónoma de Buenos Aires (Argentina).

Muestra: No probabilística, intencional, compuesta por 20 mujeres (entre 9 y 45 años) con diagnóstico de ST y sus respectivos controles agrupados por género, edad y nivel educativo. La muestra clínica estuvo conformada por niñas $(n=4)$ con media de edad de 10 años ( $D=.89$ ), adolescentes $(n=4)$ con media de edad de 16 años $(D=1.70)$ y adultos $(n=12)$ con media de edad de 26 años ( $D=8.56$ ). Por su parte, la muestra control estuvo conformada por niñas (n =4) con media de edad de 10 años ( $D=1.26$ ), adolescentes $(n=4)$ con media de edad de 16 años $(D=1.29)$ y adultos $(n=12)$ con media de edad de 26 años ( $D=7.21$ ).

La muestra se obtuvo a partir de convenios establecidos por el Grupo de Investigación Comportamiento humano, genética y ambiente (radicado en el Instituto de Psicología Básica, Aplicada y Tecnología [IPSIBAT], Universidad Nacional de Mar del Plata), con los principales centros de salud referentes en la atención del 
síndrome de Mar del Plata y Ciudad de Buenos Aires (Argentina). Los controles se seleccionaron de diferentes instituciones educativas (primarias, secundarias, terciarias y de formación laboral) públicas y privadas de la ciudad de Mar del Plata. La participación fue voluntaria y sujeta al consentimiento informado en el caso de las mujeres adultas, y el asentimiento personal en el caso de las niñas y adolescentes junto al consentimiento informado de los padres. Los controles se realizaron con la presencia de dos testigos del comité de ética de los centros de salud, quienes constataron la comprensión de las características de la investigación, la participación y los derechos de las participantes.

Como criterios de inclusión del grupo clínico se consideraron: 1) edad igual o superior a nueve años; no se incluyeron niñas con edad inferior a nueve años por considerarse la edad mínima para la aplicación de las tareas; 2) confirmación diagnóstica a través de un análisis cariotípico; 3 ) poseer, en el caso de las niñas y adolescentes, escolaridad regular; para ello se consideró como criterio de inclusión/ exclusión la ausencia de dificultades en lectoescritura, aspecto que se consideró indagando la historia escolar; 4) ausencia de patologías psiquiátricas; y 5) asistencia a los centros de salud con los cuales se realizaron los convenios.

Por otro lado, como criterios de inclusión del grupo control se consideraron: 1) edad igual o superior a nueve años; 2) presentar el mismo nivel educativo que las participantes con diagnóstico de ST; 3) poseer en el caso de las niñas y adolescentes escolaridad regular; 4) ausencia de patologías psiquiátricas; 5) asistencia a los centros educativos con los cuales se realizaron los convenios; y 6) comprensión y firma de asentimientos y consentimientos informados.

\section{Instrumentos de evaluación}

Para evaluar el aspecto cognitivo de la TdM, se administró la adaptación al español (Aguilar, Urquijo, Zabala y López, 2014) de la prueba Historias Extrañas de Happé (White, Hill, Happé y Frith, 2009) que evalúa malentendido, persuasión, doble mentira (doble bluff) y mentira piadosa (coeficiente de correlación intraclase: .89). La prueba consta de 24 historias sobre situaciones de la vida cotidiana donde las expresiones de una persona no deben ser comprendidas en su sentido literal, sino contemplando una determinada motivación subyacente. La resolución correcta implica identificar precisamente la intención que subyace a la expresión no literal de un personaje. Las respuestas obtenidas se codificaron a partir de una escala de cero a dos (0 para respuesta incorrecta, 1 para respuesta parcial o implícitamente correcta [error realista] y 2 para respuesta completamente correcta [reconocimiento del sentido no literal]). Las 24 historias han sido divididas en tres grupos (White et al., 2009). Durante la realización de la tarea se midieron los tiempos de reacción (registro exacto del tiempo transcurrido entre la realización de la pregunta que sigue a la historia y el principio de la respuesta) de cada participante.

Con el objetivo de evaluar el aspecto afectivo de la TdM, se utilizó la versión en español del Test de las miradas (Baron-Cohen, Wheelwright, Hill, Raste y Plumb, 2001). Esta prueba consta de una serie de fotografías en las que se observan miradas de hombres y mujeres que expresan un sentimiento. Su objetivo es evaluar el déficit sutil en la comprensión social en adultos con inteligencia normal, a través de la capacidad de lectura de la mente a partir de la región de los ojos. La tarea pretende determinar si el participante puede ponerse en la mente de la otra persona y sintonizar con su estado mental (Baron-Cohen et al., 2001). Este test presenta dos versiones, la versión niños y la versión adultos. La versión niños consta de 28 fotografías con cuatro opciones de respuesta, el niño debe elegir la palabra que mejor designa lo que la persona (representada por una fotografía de la mirada) está pensando o sintiendo. La respuesta correcta recibe 1 punto mientras que la incorrecta, 0 . La versión para adultos presenta las mismas características, pero 
aumenta la cantidad de estímulos a 36 . Con respecto a las propiedades psicométricas de este instrumento, solo reportan datos de confiabilidad algunas versiones, como la publicada por Vellante et al. (2013), con un coeficiente alfa de .60 .

En cuanto a la ME, se valoraron dos aspectos de la ME (de contenido y de la fuente) mediante dos tareas:

1) Para valorar la ME verbal de contenido se utilizó el test de Aprendizaje Verbal EspañaComplutense en sus dos versiones: TAVEC (versión adultos), de Benedet y Alejandre (1998), adaptación española del California Verbal Learning Test (CVLT, Delis, Kramer, Kaplan y Ober, 1987) en adultas con edades comprendidas entre 17 y 60 años, y TAVECI (versión niños) de Benedet y Alejandre (2001), en niñas y adolescentes con edades comprendidas entre 6 y 16 años. El TAVEC y el TAVECI son pruebas de evaluación de la memoria episódica y de la capacidad de aprendizaje (Benedet y Alejandre, 2014; Romero-Vanegas, Valencia-Marín, Aguirre-Acevedo, Buschke y Lopera, 2010). El TAVEC (test completo) presenta una fiabilidad estimada de .81 (error típico: 4.98). El TAVECI presenta una fiabilidad estimada entre .92 y .95 , para los distintos grupos de edad. Estos instrumentos constan de una lista de aprendizaje (lista A), una lista de interferencia (lista B) y una lista de reconocimiento. La prueba comienza con el aprendizaje auditivo verbal de las palabras de la lista A que se presentan a través de cinco ensayos. Tras el quinto ensayo de la lista A, se lee la lista B de interferencia y la persona debe recordar los elementos que la componen. Inmediatamente después de concluido el ensayo de la lista B se implementa una prueba de recuerdo libre a corto plazo de las palabras de la lista A, seguida de una prueba de recuerdo con claves semánticas a corto plazo en la que se mencionan una a una las cuatro categorías y se solicita el recuerdo de los elementos de la lista A incluidos en cada una de ellas. Pasados 20 minutos se aplica la prueba de recuerdo libre a largo plazo de la lista A y posteriormente, la prueba de recuerdo con claves semánticas a largo plazo. Finalmente, se administra la lista de reconocimiento.

Los índices que se tuvieron en consideración para valorar la ME verbal fueron los siguientes (Solcoff, 2011): a) Aciertos en la lista de reconocimiento, que representa el número de palabras de la lista A identificadas correctamente en la lista de reconocimiento. Se corresponde con un índice de recuperación ya que refleja la capacidad de identificar correctamente los ítems presentados, al ser nombrados, cuando se encuentran con otros ítems no presentados, apelando a una memoria de tipo asociativa (pasiva). Este índice se trabajará conjuntamente con el índice de sesgo, que indica hasta qué punto las respuestas de la persona en la prueba de reconocimiento han estado determinadas por una tendencia a responder sí o no; b) Recuerdo libre largo plazo, que indica la cantidad de palabras que recuerda de la lista presentada (A) sin ayuda de claves semánticas; c) Comparación de aciertos en la lista de reconocimiento con las palabras recordadas en la prueba de recuerdo libre a largo plazo, que otorga información acerca de la mayor o menor dificultad de la persona para recuperar libremente información del almacén permanente, frente a su capacidad de reconocer esa información cuando se le presenta mezclada con otra; $y$ d) Comparación de aciertos en la lista de reconocimiento con las palabras recordadas en la prueba de recuerdo con claves a largo plazo, que brinda información acerca de hasta qué punto y cómo la persona utiliza esas ayudas externas en vistas a encontrar la información deseada. Los índices 3 y 4 corresponden a procesos de memoria estratégica (activa). Al ser índices de comparación se aplica la siguiente fórmula:

\section{Puntaje directo del valor 2 - Puntaje directo del valor $1 \times 100=$ Puntaje directo valor 1}


2) Para valorar la ME de la fuente se aplicó una tarea basada en el paradigma experimental aplicado por Jacoby (1991), la cual implica reconocer el estímulo propiamente dicho, además de los elementos del contexto en el que se presentó. En esta tarea se presenta a la persona dos listas sucesivas de palabras, una oral (leída por el evaluador) y otra escrita (proyectada en una computadora). Estas listas permiten realizar dos tipos de tareas de reconocimiento: exclusión e inclusión. En la condición de exclusión, se pide a la persona que realice una recolección contextual, pues se le solicita que identifique solo las palabras de la lista oral. En la condición de inclusión se requiere el reconocimiento de las palabras de cualquiera de las dos listas. Por lo tanto, las probabilidades de acierto de la condición de inclusión se basan tanto en la aceptación de ítems de la lista oral como de la lista escrita. Se supone que el reconocimiento de una palabra de la lista escrita, en la condición de exclusión, indica que la persona ha olvidado el contexto donde se presentó esa palabra y que la respuesta de reconocimiento está basada en la familiaridad. El procedimiento se basa en el supuesto de que si una persona es capaz de recordar en qué lista fue presentado un ítem, también será capaz de incluir o excluir ese ítem según las instrucciones. La capacidad de exclusión es proporcionada por el proceso de recolección capaz de producir respuestas selectivas, ya que la familiaridad no es capaz de contribuir en ese tipo de tareas debido a que su fuerza es relativamente similar para los ítems de ambas listas y probablemente no es suficiente para discriminar los ítems de cada lista. El orden de presentación de las listas y de las pruebas (inclusión-exclusión) se aleatoriza para cada participante. Los índices que se tuvieron en consideración fueron los siguientes: 1) El índice de inclusión: cantidad de palabras a las que respondió Sí que pertenecen a las listas A y B; 2) El índice de omisiones en inclusión: cantidad de palabras a las que respondió No cuando debería haber respondido Sí, es decir cuando perte- necen a las listas A y B; 3) El índice de errores en inclusión: cantidad de palabras a las que respondió Sí cuando debería haber respondido No, es decir cuando no pertenecen a ninguna de las dos listas; 4) El índice de exclusión: cantidad de palabras a las que respondió Sí cuando debería haber respondido No, sin considerar distractores, es decir cuando son de la lista que se le presentó de manera escrita; 5) El índice de omisiones en exclusión; cantidad de palabras a las que dijo No cuando debería haber dicho Sí, es decir cuando pertenecen a la lista que se le presentó de manera oral; 6) El índice de errores en exclusión: cantidad de palabras a las que respondió Sí cuando debería haber respondido No, considerando los distractores solamente, es decir cuando no pertenecen a ninguna de las listas A y B; 7) El índice de recolección: resultado de la fórmula: inclusión-exclusión; y 8) el índice de familiaridad: Resultado de la fórmula: exclusión / (1 - recolección).

\section{Procedimiento}

Para la conformación del grupo clínico se dispuso de convenios específicos vigentes entre la Facultad de Psicología (Universidad Nacional de Mar del Plata) y centros de salud que atienden a mujeres con diagnóstico de ST. A través de los médicos endocrinólogos de dichos centros se contactó a las mujeres interesadas en participar de la investigación, solicitándoles en el caso de las adultas, la firma del consentimiento informado y en el caso de las niñas y adolescentes, la firma de consentimiento de sus padres y su asentimiento. Para la conformación del grupo control se utilizaron convenios específicos vigentes entre la Facultad de Psicología (Universidad Nacional de Mar del Plata) e instituciones educativas y laborales de la ciudad. Se establecieron contactos con las mujeres interesadas, agrupadas por edad, sexo y nivel educativo con el grupo clínico, y se solicitó la firma de los consentimientos informados y asentimientos. La batería de técnicas que 
evalúan TdM y ME se administró de forma individual en dos encuentros de una hora cada uno, con una frecuencia semanal. Las evaluaciones se realizaron en las instalaciones del IPSIBAT, perteneciente a la Facultad de Psicología (Universidad Nacional de Mar del Plata). La realización del estudio respetó normas éticas consideradas por las instituciones intervinientes, los derechos civiles de los participantes y la Declaración de Helsinki. Asimismo, los procedimientos implementados fueron aprobados por el Comité de Ética del Programa Temático Interdisciplinario en Bioética, dependiente de la Secretaría de Ciencia y Técnica de la Universidad Nacional de Mar del Plata.

\section{Análisis de datos}

Para analizar los aspectos cognitivo $\mathrm{y}$ afectivo de la TdM y la ME en mujeres con diagnóstico de ST, los datos obtenidos fueron sometidos a un análisis estadístico descriptivo (medias, desvíos, frecuencias y porcentajes) y para comparar su desempeño con mujeres con desarrollo típico se aplicaron pruebas estadísticas de comparación de grupos. Debido a que algunas de las variables analizadas no presentan una distribución normal en la población abordada de acuerdo al Test de Levene, se utilizó en esos casos la prueba no paramétrica $U$ de Mann-Whitney, alternativa a la prueba $t$ de Student cuando no se cumple el supuesto de normalidad. También se calculó el tamaño del efecto, a través de la prueba Delta de Cliff para pruebas no paramétricas, donde un tamaño de efecto de +1.0 o -1.0 indica la ausencia de superposición entre los dos grupos, mientras que 0.0 indica que las distribuciones de grupos se superponen completamente. Cuando se obtiene un valor de $p$ significativo, se espera que el tamaño del efecto asociado esté cerca de +1.0 o -1.0 debido a que la diferencia entre los grupos es importante (Macbeth, Razumiejczyk y Ledesma, 2011). Para analizar las relaciones de los aspectos cognitivos y afectivos de la TdM con la capacidad de ME en las mujeres con diagnóstico de ST, se transformaron las puntuaciones brutas a puntajes $z$ para comparar grupo clínico y control. Posteriormente, se realizaron análisis multivariados para controlar el efecto de la edad en las variables. Por último, se efectuaron análisis de correlaciones no paramétricas (Rho de Spearman), con la finalidad de observar posibles relaciones entre las variables.

\section{Resultados}

En primer lugar, la población clínica se consideró como un único grupo (grupo clínico), ya que la aplicación de la prueba $U$ de Mann-Whitney a las distintas variables bajo estudio mostró la ausencia de diferencias significativas entre los diferentes grupos etarios (niñas, adolescentes y adultas).

A continuación, se presentan los análisis no paramétricos de comparación de dos muestras independientes. La Tabla 1 muestra los resultados de análisis descriptivos e inferenciales para cada una de las dimensiones de TdM.

Tabla 1

Análisis descriptivos e inferenciales entre muestra clínica y control en las dimensiones de TdM.

\begin{tabular}{c|c|c|c|c|c} 
& $\begin{array}{c}\text { Grupo clínico } \\
(\mathbf{N}=\mathbf{2 0}) \boldsymbol{M}(\mathbf{D S})\end{array}$ & $\begin{array}{c}\text { Grupo control } \\
(\mathbf{N}=\mathbf{2 0}) \boldsymbol{M}(\mathbf{D S})\end{array}$ & $\begin{array}{c}\text { U de Mann- } \\
\text { Whitney }\end{array}$ & $\boldsymbol{Z}$ & $\begin{array}{c}\boldsymbol{D} \text { de } \\
\text { Cliff }\end{array}$ \\
\hline TdM cognitiva & $-.57(.92)$ & $.51(.69)$ & $86.00 *$ & -3.66 & .02 \\
TdM afectiva & $-.25(1.29)$ & $.21(.51)$ & 187.00 & -1.28 & -.39
\end{tabular}

$* \mathrm{p} \leq .01$ 
Los resultados permiten observar la existencia de diferencias estadísticamente significativas en TdM cognitiva entre grupo clínico y grupo control (menor rendimiento en el grupo clínico), aunque con una magnitud del efecto baja. Asimismo, en la tarea que evalúa el componente afectivo de la TdM no se observaron diferencias significativas entre los grupos.

En la Tabla 2 se presentan los resultados de análisis descriptivos y pruebas de diferencias, y magnitud del efecto entre muestra clínica y control para los indicadores de la ME de contenido.

Tabla 2

Análisis descriptivos e inferenciales entre muestra clínica y control en los índices de ME de contenido (TAVEC-TAVECI).

\begin{tabular}{c|c|c|c|c|c} 
& $\begin{array}{c}\text { Grupo clínico } \\
\text { (N= 20) } \boldsymbol{M} \text { (DS) }\end{array}$ & $\begin{array}{c}\text { Grupo control } \\
\text { (N= 20) } \boldsymbol{M}(\mathbf{D S})\end{array}$ & $\begin{array}{c}\text { U de Mann- } \\
\text { Whitney }\end{array}$ & $\boldsymbol{Z}$ & $\boldsymbol{D}$ de Cliff \\
\hline AC & $-.15(1.22)$ & $.46(.61)$ & 92.50 & -1.39 & -.28 \\
Sesgo & $-.06(1.79)$ & $.00(.00)$ & 120.00 & -.38 & -.06 \\
RL-LP & $1.08(.04)$ & $.81(.47)$ & $77.00^{*}$ & -1.95 & -.40 \\
AC/RL-LP & $-1.31(1.52)$ & $.78(.44)$ & $12.00^{*}$ & -4.54 & -.90 \\
AC/RCl-LP & $-1.31(1.48)$ & $.71(.60)$ & $17.50^{*}$ & -4.31 & -.86
\end{tabular}

Notas: AC: Aciertos en la lista de reconocimiento; RL-LP: Recuerdo libre largo plazo; AC/RL-LP: Aciertos en la lista de reconocimiento frente a recuerdo libre a largo plazo; AC/RCl-LP: Aciertos en la lista de reconocimiento frente a recuerdo con claves a largo plazo. ${ }^{*} \mathrm{p} \leq .01$

Los resultados permiten observar la existencia de diferencias estadísticamente significativas entre los grupos en los siguientes indicadores de ME de contenido: RL-LP, AC/RL-LP, AC/RCl-LP. La magnitud del efecto es negativa evidenciando un desempeño menor del grupo clínico en relación al grupo control, cuando se comparan los aciertos en reconocimiento con otros indicadores que suponen una recuperación espontánea o guiada de la información.

La Tabla 3 presenta los resultados de las pruebas de diferencias de medias y magnitud del efecto entre muestra clínica y control para los indicadores de ME de la fuente.

\section{Tabla 3}

Análisis descriptivos e inferenciales entre muestra clínica y control en los indicadores de ME de la fuente.

\begin{tabular}{|c|c|c|c|c|c|}
\hline & $\begin{array}{c}\text { Grupo clínico } \\
(\mathrm{N}=\mathbf{2 0}) M(\mathrm{DS}) \\
\end{array}$ & $\begin{array}{c}\text { Grupo control } \\
(\mathbf{N}=\mathbf{2 0}) M(\mathrm{DS}) \\
\end{array}$ & $\begin{array}{c}\text { U de Mann- } \\
\text { Whitney } \\
\end{array}$ & $Z$ & $D$ de Cliff \\
\hline Inclusión & $-.31(1.11)$ & $.18(.84)$ & 105.50 & -.85 & -.17 \\
\hline Errores en inclusión & $.14(1.17)$ & $-.25(.80)$ & 101.50 & -1.01 & .20 \\
\hline Omisiones en inclusión & $.38(1.18)$ & $-.18(.71)$ & 99.50 & -1.08 & .22 \\
\hline Exclusión & $.26(1.14)$ & $-.42(.69)$ & $70.00^{*}$ & -2.20 & .45 \\
\hline Errores Exclusión & $.25(1.11)$ & $-.17(1.06)$ & 80.50 & -1.83 & .38 \\
\hline Omisiones Exclusión & $.49(1.04)$ & $-.30(.84)$ & $67.00 *$ & -2.31 & .48 \\
\hline Familiaridad & $-.25(1.17)$ & $.33(.64)$ & 83.00 & -1.70 & -.35 \\
\hline Recolección & $-.39(1.07)$ & $.40(.82)$ & $77.00^{*}$ & -1.93 & -.40 \\
\hline
\end{tabular}


Los resultados permiten observar la existencia de diferencias estadísticamente significativas entre los grupos en los siguientes indicadores de ME de la fuente: exclusión, omisiones en exclusión y recolección. Las puntuaciones positivas en el Delta de Cliff en exclusión y omisiones en exclusión indican un número mayor de errores en estos índices para el grupo de mujeres con diagnóstico de
ST, evidenciando un desempeño inferior en la identificación del contexto. A su vez, el valor negativo en el índice de recolección señala que las mujeres con diagnóstico de ST presentaron un desempeño inferior en la recuperación del contexto.

La Tabla 4 presenta correlaciones al interior de las mujeres con diagnóstico de ST, realizando un análisis intragrupo.

Tabla 4

Relaciones entre las dimensiones de la TdMy ME.

\begin{tabular}{cc|c|c} 
& & TdM cognitiva & TdM afectiva \\
\hline & AC & .30 & .25 \\
ME verbal de & Sesgo & .17 & .15 \\
contenido & RL-LP & $.51^{*}$ & $.55^{*}$ \\
& AC/RCl-LP & $.55^{*}$ & $.53^{*}$ \\
& AC/RL-LP & $.53^{*}$ & $.50^{* *}$ \\
\hline \multirow{3}{*}{$\begin{array}{c}\text { ME verbal de la } \\
\text { fuente }\end{array}$} & Errores en exclusión & -.41 & $-.53^{*}$ \\
& Omisiones en & .13 & -.14 \\
& exclusión & -.02 & -.24 \\
& Recolección & .39 & $.60^{* *}$
\end{tabular}

Notas: AC: Aciertos en la lista de reconocimiento; RL-LP: Recuerdo libre largo plazo; AC/RL-LP: Aciertos en la lista de reconocimiento frente a recuerdo libre a largo plazo; AC/RCl-LP: Aciertos en la lista de reconocimiento frente a recuerdo con claves a largo plazo. $* * \mathrm{p} \leq .01 . * \mathrm{p} \leq .05$.

Se observan correlaciones positivas medias entre las dimensiones de la TdM y el desempeño en procesos de recuperación activa $\mathrm{y}$ correlaciones medias positivas entre TdM afectiva y el proceso de recolección. Por otro lado, las correlaciones entre TdM afectiva y exclusión son correlaciones medias negativas.

\section{Discusión}

En primera instancia, los resultados presentados aportan evidencia a la hipótesis que las mujeres con diagnóstico de ST presentan dificultades de carácter general en la TdM con un rendimiento diferencial en los aspectos cognitivos y afectivos, siendo menor el desempeño en la TdM cognitiva.
Asimismo, se observan dificultades en la ME verbal de contenido y la memoria verbal de la fuente, particularmente en relación con la recuperación del contexto. En lo que respecta a ME de contenido, si bien las mujeres con diagnóstico de ST evidencian una capacidad normal de reconocimiento, cuando se relaciona el número de aciertos en la lista de reconocimiento con la cantidad de elementos recuperados en la prueba de recuerdo libre a largo plazo (AC/RL-LP), así como con la cantidad de elementos recuperados en la prueba a largo plazo con claves (AC/RCl-LP), se encuentra mayor dificultad para recuperar libremente $\mathrm{O}$ en forma guiada (mediante claves semánticas) la información del almacén permanente. Estos resultados podrían indicar que a las mujeres 
con diagnóstico de ST les resulta más sencilla la identificación de una información cuando se le presenta mezclada con otra, tendiendo a necesitar mayor ayuda externa para recuperar información. Esto podría vincularse a una falta de consistencia en el recuerdo, con fallas en la capacidad de usar sistemáticamente estrategias adecuadas de recuperación activa de la información.

En relación a la ME de la fuente también se evidencia una mayor dificultad en la identificación contexto, presentando menores rendimientos en los índices relacionados con los procesos de recolección (exclusión), pero no con los procesos de familiaridad (inclusión). Este desempeño diferencial implicaría dificultades a la hora de identificar el contexto de diferenciación.

Con respecto a la vinculación de los procesos de TdM y ME verbal en las mujeres con diagnóstico de ST y en línea con numerosas investigaciones (Buckner y Carroll, 2007; Lind et al., 2013; Moreau et al., 2013; Naito, 2003; Nandrino et al., 2014; Perner, 2000; Solcoff, 2011; Spreng y Mar, 2012), los resultados hallados evidencian relaciones entre ambos procesos. En este sentido, se encontraron correlaciones positivas medias entre las dimensiones cognitivas y afectivas de la TdM con los indicadores de ME verbal de contenido que presentaron menor rendimiento en la población clínica: recuerdo libre a largo plazo, aciertos en reconocimiento frente a recuerdo libre a largo plazo y aciertos en reconocimiento frente a recuerdo con claves a largo plazo. Dichas correlaciones indican una asociación positiva entre las dimensiones de $\mathrm{TdM}$ con procesos de recuperación activa de la ME. Es decir, las mujeres con diagnóstico de ST presentan problemas para recuperar información en forma espontánea o guiada por claves, lo que daría cuenta de dificultades en forjar estrategias que permitan organizar la información y evocar la representación sin ayudas contextuales.

Las asociaciones entre la ME verbal de la fuente y la TdM afectiva indicarían dificultades en el reconocimiento de una experiencia subjetiva y, por ende, a en la reexperiencia de esa vivencia. La mayor especificidad en la identificación del contexto implica necesariamente la posibilidad de reexperimentación subjetiva de una vivencia donde el self y sus estados mentales propios se tornan centrales, así como la diferenciación con los estados mentales ajenos, siendo este último un aspecto troncal en el funcionamiento de la TdM (Lind et al., 2013; Solcoff, 2011). Vogeley (2017) señala que para comprender las experiencias de otros se requiere de tres aspectos fundamentales: comunicación verbal y no verbal, conocimiento previo y contexto situacional. Este último es el que permitiría forjar huellas de mayor pregnancia que puedan ser recuperadas como parte de las experiencias subjetivas de la persona.

$\mathrm{Si}$ se analizan los resultados obtenidos considerando que la TdM emerge con anterioridad a la ME (Naito, 2003; Perner, 2000; Solcoff, 2011) y posibilita, a través de la capacidad de metarrepresentación, el desdoblamiento y viaje mental que la ME implica, se podría hipotetizar que los problemas observados en las mujeres con diagnóstico de ST en la ME verbal podrían relacionarse con dificultades previas en TdM. De esta manera, se contribuiría con evidencias empíricas a la hipótesis que sugiere que las anomalías en la estructura cerebral que presentan las mujeres con dicho diagnóstico, podrían provocar disfunciones tempranas en el desarrollo de la TdM, que a su vez darían lugar a dificultades de la ME verbal. En esta línea, Buckner y Carroll (2007) proponen el concepto de autoproyección que supone que los procesos de previsión episódica (ligada a la ME en cuanto a proyección temporal), TdM, memoria prospectiva y navegación son parte del mismo constructo. La red neuronal requerida para la proyección hacia otro tiempo (pasado o futuro) o perspectiva (de otra persona o de otro punto de vista) es común a estos procesos y diferenciada de otras tareas que requieren resolución de problemas o simple imaginación (Lind et al., 2013; Vázquez-Echeverría, 2015). 
Lo expuesto contribuye a lograr una mayor comprensión del perfil neuropsicológico de las mujeres con diagnóstico de ST. La información brindada resulta vinculante al campo de acción de la neuropsicología y a los profesionales que integran equipos interdisciplinarios de salud, al brindar un soporte empírico que permite valorar la estrecha relación entre los procesos estudiados. Dicho soporte teórico podría servir de insumo para el diseño de estrategias clínicas y educativas que tiendan a fomentar las habilidades en TdM y ME, considerando que la estimulación de la TdM en mujeres con diagnóstico de ST podría tener injerencia en el funcionamiento de la $\mathrm{ME}$, sobre todo en lo que respecta al recuerdo del contexto.

Finalmente, cabe considerar que, si bien el presente trabajo ha dado cuenta de la relación entre la TdM y la ME verbal en mujeres con diagnóstico de $\mathrm{ST}$, el número pequeño de la muestra limita la generalización de las conclusiones. Sería sustancial la profundización del análisis de este vínculo en diferentes etapas del ciclo vital de esta población (niñas, adolescentes y adultas), hecho que permitiría obtener una descripción completa y ajustada de relación de ambos procesos durante el desarrollo. Por este motivo, se considera relevante incorporar futuras líneas de investigación que incluyan un mayor número muestral para cada etapa evolutiva y modelos estadísticos explicativos, que permitan establecer la relación direccional entre ambas variables. Asimismo, resulta interesante pensar un diseño de investigación pre y post, con protocolos de intervenciones para mejorar el funcionamiento de TdM y medidas de evaluación pre y post test de $\mathrm{ME}$, que permita profundizar el conocimiento de la relación entre ambos procesos.

\section{Referencias bibliográficas}

Aguilar, M.J., Urquijo, S., Zabala, M.L. y López, M. (2014). Aportes empíricos a la validación y adaptación al español de la tarea mentalista de Historias Extrañas. Revista Argentina de Ciencias del Comportamiento, 6(2), 1-10.
Aguilar, M.J., Zabala, M.L., López-Morales, H., Urquijo, S. y López, M. (2016). La teoría de la mente como proceso mediador del funcionamiento social. Evaluación en el Síndrome de Turner. Revista Argentina de Ciencias del Comportamiento, 8(3), 40-48. E-ISSN: 1852-4206

Allen, T.A. y Fortin, N.J. (2013). The evolution of episodic memory. Proceedings of the National Academy of Sciences, 110(2), 10379-10386. https://doi.org/10.1073/pnas.1301199110

Anaki, D., Zadikov-Mor, T., Gepstein, V. y Hochberg, Z. (2016). Face perception in women with Turner syndrome and its underlying factors. Neuropsychologia, 90, 274-285. https://doi. org/10.1016/j.neuropsychologia.2016.08.024

Baron-Cohen, S., Wheelwright, S., Hill, J., Raste, Y. y Plumb, I. (2001). The "Reading the mind in the eyes" test revised version: a study with normal adults and adults with Asperger syndrome or high-functioning autism. Journal of Child Psychology and Psychiatry, 42(2), 241-251. https://doi.org/10.1111/14697610.00715

Benedet, M.J. y Alejandre, M.A (1998). Test de aprendizaje verbal España Complutense. Madrid: TEA Ediciones. S.A.

Benedet, M.J. y Alejandre, M.A (2001). Test de aprendizaje verbal España Complutense infantil. Madrid: TEA Ediciones. S.A.

Benedet, M.J. y Alejandre, M.A. (2014). TAVEC. Test de Aprendizaje Verbal España-Complutense. Madrid: TEA Ediciones.

Buckner, R.L. y Carroll, D.C. (2007). Self-projection and the brain. Trends in Cognitive Sciences, 11(2), 49-57. https://doi.org/10.1016/j. tics.2006.11.004

Cooper, E., Greve, A. y Henson, R.N. (2017). Assumptions behind scoring source versus item memory: Effects of age, hippocampal lesions and mild memory problems. Cortex, 91, 297-315. https://doi.org/10.1016/j. cortex.2017.01.001

Delis, D.C., Kramer, J.H., Kaplan, E. y Ober, B.A. (1987). California Verbal Learning Test. San Antonio: The Psychological Corporation. https://doi.org/10.1037/t15072-000 
Duval, C., Piolino, P., Bejanin, A., Eustache, F. y Desgranges, B. (2011). Age effects on different components of theory of mind. Consciousness and cognition, 20(3), 627-642. https://doi. org/10.1016/j.concog.2010.10.025

Eichenbaum, H. (2017). Prefrontal-hippocampal interactions in episodic memory. Nature Reviews Neuroscience, 18(9), 547-558. https:// doi.org/10.1038/nrn.2017.74

González-Cuenca, A.M., Barajas-Esteban, C., Linero-Zamorano, M.J. y Quintana-García, I. (2008). Deficiencia auditiva y teoría de la mente. Datos para la reflexión y la intervención. Revista de Logopedia, Foniatría y Audiología, 28(2), 99-116. https://doi.org/10.1016/ s0214-4603(08)70049-4

Granger, A., Zurada, A., Zurada-Zielińska, A., Gielecki, J. y Loukas, M. (2016). Anatomy of Turner Syndrome. Clinical Anatomy, 29(5), 638-642. https://doi.org/10.1002/ca.22727

Hernández-Sampieri, R., Fernández-Collado, C. y Baptista-Lucio, P. (2014). Metodología de la Investigación. México: Mc Graw Hill.

Hong, D., Dunkin, B. y Reiss, A. (2011). Psychosocial functioning and social cognitive processing in girls with Turner Syndrome. Journal of Developmental \& Behavioral Pediatrics, 32(7), 512-520. https://doi.org/10.1097/ dbp.0b013e3182255301

Hughes, C. y Devine, R.T. (2015). A Social Perspective on Theory of Mind. En R.M. Lerner (Ed.), Handbook ofChild Psychology and Developmental Science (1-46). New Jersey: Wiley. https://doi.org/10.1002/9781118963418.childpsy3 314

Jacoby, L. (1991). A process dissociation framework: Separating automatic from intentional uses of memory. Journal of Memory and Language, 30(5), 513-541. https://doi. org/10.1016/0749-596x(91)90025-f

Kalbe, E., Schlegel, M., Sack, A., Nowak, D., Dafotakis, M., Bangard, C., ... y Kessler, J. (2010). Dissociating cognitive from affective theory of mind: A TMS study. Cortex, 46(6), 769-780. https://doi.org/10.1016/j. cortex.2009.07.010

Klein, K.O., Rosenfield, R., Santen, R.J., Gawlik,
A., Backeljauw, P., Gravholt, C.H., ... y Mauras, N. (2018). Estrogen Replacement in Turner Syndrome: Literature Review and Practical Considerations. The Journal of Clinical Endocrinology \& Metabolism, 103(5), 1790-1803. https://doi.org/10.1210/jc.2017-02183

Lepage, J.F., Dunkin, B., Hong, D.S. y Reiss, A.L. (2011). Contribution of Executive Functions to Visuospatial Difficulties in Prepubertal Girls With Turner Syndrome. Developmental Neuropsychology, 36(8), 988-1002. https:// doi.org/10.1080/87565641.2011.584356

Lesniak-Karpiak, K., Mazzocco, M. y Ross, J. (2003). Behavioral assessment of social anxiety in females with Turner or Fragile X Syndrome. Journal of Autism and Developmental Disorders, 33(1), 55-67. https://doi. org/10.1023/a:1022230504787

Lind, S.E., Williams, D.M., Raber, J., Peel, A. y Bowler, D.M. (2013). Spatial Navigation Impairments Among Intellectually High-Functioning Adults With Autism Spectrum Disorder: Exploring Relations With Theory of Mind, Episodic Memory, and Episodic Future Thinking. Journal of Abnormal Psychology, 122(4), 1189-1199. https://doi.org/10.1037/a0034819

Macbeth, G., Razumiejczyk, E. y Ledesma, R. (2011). Cliff's Delta Calculator: A non-parametric effect size program for two groups of observations. Universitas Psychologica, 10(2), 545-555. https://doi.org/10.11144/javeriana. upsy10-2.cdcp

Marzelli, M., Hoeft, F., Hong, D. y Reiss, A. (2011). Neuroanatomical Spatial Patterns in Turner Syndrome. Neuroimage, 55(2), 439-447. https://doi.org/10.1016/j.neuroimage.2010.12.054

Mauger, C., Lancelot, C., Roy, A., Coutant, R., Cantisano, N. y Le Gall, D. (2018). Executive Functions in Children and Adolescents with Turner Syndrome: A Systematic Review and Meta-Analysis. Neuropsychology Review, 28(2), 188-215. https://doi.org/10.1007/ s11065-018-9372-x

Mazzocco, M. (2006). The cognitive phenotype of Turner Syndrome: Specific learning disabilities. International Congress Series, 1298, 83-92. 
https://doi.org/10.1016/j.ics.2006.06.016

Moreau, N., Viallet, F. y Champagne-Lavau, M. (2013). Using memories to understand others: The role of episodic memory in theory of mind impairment in Alzheimer disease. Ageing Research Reviews, 12(4), 833-839. https://doi. org/10.1016/j.arr.2013.06.005

Moses, L.J., Carlson, S.M. y Sabbagh, M.A. (2005). On the Specificity of the Relation Between Executive Function and Children's Theories of Mind. En W. Schneider, R. Schumann-Hengsteler y B. Sodian (Eds.), Young Children's Cognitive Development: Interrelationships among Executive Functioning, Working Memory, Verbal Ability, and Theory of Mind. (131-146). New York: Psychology Press. https://doi.org/10.4324/9781410612007

Naito, M. (2003). The relationship between theory of mind and episodic memory: Evidence for the development of autonoetic consciousness. Journal of Experimental Child Psychology, 85(4), 312-336. https://doi.org/10.1016/ s0022-0965(03)00075-4

Nandrino, J.-L., Gandolphe, M.-C., Alexandre, C., Kmiecik, E., Yguel, J. y Urso, L. (2014). Cognitive and affective theory of mind abilities in alcohol-dependent patients: The role of autobiographical memory. Drug and Alcohol Dependence, 143, 65-73. https://doi. org/10.1016/j.drugalcdep.2014.07.010

Perner, J. (2000). Memory and Theory of Mind. En E. Tulving y F. Craik (Eds.), The Oxford Handbook of Memory (297-312). Oxford: Oxford University Press. ISBN: 0-19-512265-8

Rivière, A. (1996). La mirada mental: desarrollo de las capacidades cognitivas interpersonales. Buenos Aires: Aique.

Romero-Vanegas, S.J., Valencia-Marín, C.M., Aguirre-Acevedo, D.C., Buschke, H. y Lopera, F. (2010). Alteraciones de la memoria episódica verbal en fases preclínica y temprana de la enfermedad de Alzheimer familiar precoz por mutación E280A en PS1. Acta Neurológica Colombiana, 26(4), 178-194.

Sartori, M.S. y López, M.C. (2016). Habilidades sociales: Su importancia en mujeres con diagnóstico de Síndrome de Turner. Revista Lati- noamericana de Ciencias Sociales, Niñez y Juventud, 14(2), 1055-1067. https://doi.org/10. 11600/1692715x.14212160115

Sartori, M.S., Urquijo, S., López, M., Said, A. y Alchieri, J.C. (2015). Análisis de perfiles de personalidad en mujeres adultas con diagnóstico de Síndrome de Turner. Interdisciplinaria, 32(1), 73-87. https://doi.org/10.16888/ interd.2015.32.1.4

Schiller, D., Eichenbaum, H., Buffalo, E.A., Davachi, L., Foster, D.J., Leutgeb, S. y Ranganath, C. (2015). Memory and Space: Towards an Understanding of the Cognitive Map. The Journal of Neuroscience, 35(41), 13904-13911. https://doi.org/10.1523/jneurosci.2618-15.2015

Schneider, W., Schumann-Hengsteler, R. y Sodian, B. (2005). Young children's cognitive development: Interrelationships among executive functioning, working memory, verbal ability, and theory of mind. New York: Psychology Press. https://doi.org/10.4324/9781410612007

Shamay-Tsoory, S., Harari, H., Aharon-Peretz, J. y Levkovitz, L. (2010). The role of the orbitofrontal cortex in affective theory of mind deficits in criminal offenders with psychopathic tendencies. Cortex, 46(5), 668-677. https://doi. org/10.1016/j.cortex.2009.04.008

Sodian, B. (2005). Theory of Mind - The Case for Conceptual Development. En W. Schneider, R. Schumann-Hengsteler y B. Sodian (Eds.), Young Children's Cognitive Development: Interrelationships among Executive Functioning, Working Memory, Verbal Ability, and Theory of Mind (95-131). New York: Psychology Press. https://doi.org/10.4324/9781410612007

Solcoff, K. (2011). El origen de la Memoria Episódica y de Control de Fuente: Su relación con las capacidades de Teoría de la Mente (Tesis doctoral inédita). Facultad de Psicología, Universidad Autónoma de Madrid, España.

Spreng, R.N. y Mar, R.A. (2012). I remember you: A role for memory in social cognition and the functional neuroanatomy of their interaction. Brain Research, 1428, 43-50. https://doi. org/10.1016/j.brainres.2010.12.024

Tirapu-Ustárroz, J., Pérez-Sayesa, G., Ereka- 
txo-Bilbaoa, M. y Pelegrín-Valerob, C. (2007). ¿Qué es la teoría de la mente? Revista de Neurología, 44(8), 479-489. https://doi. org/10.33588/rn.4408.2006295

Tulving, E. (2002). Episodic Memory: From mind to brain. Annual Review of Psychology, 53(1), 1-25. https://doi.org/10.1146/annurev. psych.53.100901.135114

Tulving, E. (2005). Episodic memory and autonoesis: Uniquely human. En H. Terrace y J. Metcalfe (Eds.), The missing link in cognition: Origins of self-reflective consciousness (3-56). Oxford: Oxford University Press. https://doi.org/10.1093/acprof:oso/9780195161564.003.0001

Van den Hoven, A.T., Roos-Hesselink, J.W. y Timmermans, J. (2017). Turner Syndrome. Aneurysms-Osteoarthritis Syndrome, 89-101. https://doi.org/10.1016/b978-0-12-8027080.00010-7

Vargo, T., Klabunde, M., Hong, D. y Reiss, A. (2017). Visuospatial Processing and Executive Functioning in Turner Syndrome. Archives of Clinical Neuropsychology, 32(6), 667-765. https://doi.org/10.1093/arclin/acx076.118

Vázquez-Echeverría, A. (2015). La Previsión Episódica en la Etapa Pre-Escolar: Equiva- lencia entre Medidas y su Relación con los Procesos de Orientación Futura y la Teoría de la Mente. Psicologia: Reflexão e Crítica, 28(1), 157-165. https://doi.org/10.1590/16787153.201528117

Vellante, M., Baron-Cohen S., Melis, M., Marrone, M., Petretto, D., Masala, C. y Preti, A. (2013). The "Reading the Mind in the Eyes" Test: Systematic Review of Psychometric Properties and a Validation Study in Italy. Journal Cognitive Neuropsychiatry, 18(4), 326-354. https:// doi.org/10.1080/13546805.2012.721728

Vogeley, K. (2017). Two social brains: neural mechanisms of intersubjectivity. Philosophical Transactions of the Royal Society B: Biological Sciences, 372(1727), 20160245. https:// doi.org/10.1098/rstb.2016.0245

Wang, Z. y Su, Y. (2013). Age-related differences in the performance of theory of mind in older adults: A dissociation of cognitive and affective components. Psychology and Aging, 28(1), 284-291. https://doi.org/10.1037/a0030876

White, S., Hill, E., Happé, F. y Frith, U. (2009). Revisiting the Strange Stories: Revealing Mentalizing Impairments in Autism. Child Development, 80(4), 1097-1117. https://doi. org/10.1111/j.1467-8624.2009.01319.x

Recibido: 12 de abril de 2018 Aceptado: 8 de abril de 2020 